\title{
Novel endoscopic treatment strategy for early esophageal cancer in cirrhotic patients with esophageal varices
}

\author{
ZHENG-GUO XU, YONG-BING ZHAO, JIN YU, JIAN-YING BAI, EN LIU, BO TANG and SHI-MING YANG \\ Department of Gastroenterology, Xinqiao Hospital, Third Military Medical University, Chongqing 400037, P.R. China
}

Received October 18, 2018; Accepted April 17, 2019

DOI: $10.3892 / \mathrm{ol} .2019 .10532$

\begin{abstract}
The safety and efficacy of endoscopic submucosal dissection (ESD) and radiofrequency ablation for early esophageal cancer (EEC) in cirrhotic patients has not been thoroughly investigated to date. The present study aimed to establish a standard treatment strategy for EEC in cirrhotic patients with esophageal varices. Six cirrhotic patients with early flat-type EECs (high-grade intraepithelial neoplasia) on or adjacent to esophageal varices were enrolled. Esophageal varix ligation (EVL) or transjugular intrahepatic portosystemic shunt (TIPS) were used for the initial management of esophageal varices. Follow-up endoscopy was performed two months following the initial procedure. The mean longitudinal length of the lesions was $4.3 \mathrm{~cm}$ (range, $2-6 \mathrm{~cm}$ ). The average procedure time was $72.8 \mathrm{~min}$ (range, 34-135 min) and the average longitudinal length of the resected specimens was $45.6 \mathrm{~mm}$ (range, 30-90 mm). One case had a tumor-positive lateral margin with lymphovascular infiltration. Both complete and curative resection rates were $80 \%$ (4/5 lesions). Large intraoperative bleeding was detected in patients undergoing EVL compared with TIPS prior to the ESD procedure. No severe complications or mortality-associated events, including massive postoperative bleeding, perforation or hepatic failure, were observed. No recurrence and metastasis were observed during the follow-up period. The current study suggested a novel treatment strategy for EECs complicated by esophageal varices in cirrhosis with good treatment results, no neoplastic progression and an acceptable adverse event profile.
\end{abstract}

\section{Introduction}

Esophageal cancer is often diagnosed in the advanced stages of the disease (1). This highlights the requirement to

Correspondence to: Professor Shi-Ming Yang or Dr Bo Tang, Department of Gastroenterology, Xinqiao Hospital, Third Military Medical University, 183 Xinqiao Street, Chongqing 400037, P.R. China E-mail: shimingyang@yahoo.com

E-mail: atreebobo@163.com

Key words: early esophageal cancer, liver cirrhosis, esophageal varices, transjugular intrahepatic portosystemic shunt, endoscopic treatment identify patients with early esophageal cancer and to provide appropriate treatment as soon as possible. The development of techniques, including chromoendoscopy, narrow-band imaging (NBI), magnification endoscopy, confocal microscopy and spectroscopy, has facilitated the diagnosis of early superficial esophageal cancer $(2,3)$. Early esophageal cancer (EEC) refers to lesions confined to the mucosa (lamina propria and muscularis mucosa) regardless of lymph node or distant organ metastasis (4,5). Endoscopic submucosal dissection (ESD) is an established procedure for the treatment of early superficial esophageal cancer $(6,7)$. Compared with endoscopic mucosal resection, ESD provides a high en bloc resection rate $(6,7)$. Previous studies reported that esophageal ESD may be associated with adverse events, including postoperative bleeding and perforation $(8,9)$. Therefore, ESD is contraindicated in patients with lesions that occur close to the blood vessels, including esophageal varices, due to the increased risk of bleeding (10).

Patients with cirrhosis associated with superficial esophageal cancer have been increasingly reported (11-14). ESD for patients with cirrhosis may carry a higher risk of postoperative bleeding due to the low platelet count, coagulopathy and particularly due to the esophageal varices in these patients (10). Although previous case reports described the use of ESD for EEC in patients with liver cirrhosis (12), the safety and efficacy of ESD for EEC in these patients remains uncertain. The aim of the current study was to investigate the efficacy, safety and treatment flowchart of EEC in patients with cirrhosis with or without esophageal varices.

\section{Materials and methods}

Ethics statement. The current study was conducted in accordance with the Declaration of Helsinki. The study protocol was approved by the Ethics Committee of Xinqiao Hospital of Third Military Medical University (Chongqing, China). Informed consent for participation in the study and publication of the images associated with this manuscript was obtained from all patients.

Patient selection. A total of 6 male patients with cirrhosis and EEC were enrolled between February 2014 and July 2018 at the Xinqiao Hospital of Third Military Medical University (Chongqing, China). The average age was 57 years with an age range of 48-66 years old. The patients were diagnosed with cirrhosis based on ultrasonography, radiology and laboratory 
investigations. Early-stage esophageal cancer (squamous high-grade intraepithelial neoplasms and intramucosal squamous carcinomas) was diagnosed by narrow band imaging and histological biopsy prior to the ESD procedure. All patients had esophageal varices. All patients had early EEC (type $0-\mathrm{II} b$ ) overlying or distant from the esophageal varices. The location of the lesion was defined by the distance from the incision to the lesion. Endoscopic grading of esophageal varices was based on the classification defined by the Japanese Research Society of Portal Hypertension (15). Esophageal varix forms were classified as follows: F1, small straight varix; $\mathrm{F} 2$, enlarged tortuous varix that occupies less than one-third of the lumen; and F3, large coil-shaped varix that occupies more than one-third of the lumen (15). The presence of the red wale marking was described as red-sign positive.

Endoscopic variceal ligation procedure. An endoscope (cat. no. GIF-Q260J; Olympus Corporation, Tokyo, Japan) was used for the EVL procedure. A $25 \mathrm{~cm}$ overtube was backloaded over the shaft of the endoscope. Ligation was performed by two experienced endoscopists who had $>10$ years of experience. After the endoscope had entered the esophagus, the overtube was pushed forward over the shaft of the endoscope. The endoscopic ligating device was then attached to the distal end of the endoscope. Ligation was performed at $1-5 \mathrm{~cm}$ above the gastroesophageal junction. Each varix was ligated with one 1-3 rubber bands. A maximum of 10 rubber bands per patient were used for ligation.

Transjugular intrahepatic portosystemic shunt (TIPS) procedure. Ultrasonography was performed in each patient to evaluate the portal vein prior to the TIPS procedure. TIPS was performed under general anesthesia by the same experienced interventional radiologist. Briefly, vascular access was obtained through the right internal jugular vein, followed by catheterization of the right hepatic vein. Intrahepatic puncture was performed from the right hepatic vein to the right or left portal vein. Successful trans-hepatic puncture of the portal venous system was confirmed by angiography for confirmation and assessment of the puncture location. The puncture tract was dilated using an angioplasty balloon (diameter, $7 \mathrm{~mm}$ ). Subsequently, 8-mm Viatorr ${ }^{\circledR}$ covered stent-grafts covered with polytetrafluoroethylene (W.L. Gore and Associates, Newark, DE, USA) were inserted from the portal vein up to the confluence of the right hepatic vein and the inferior vena cava. Shunt placement was considered successful when portosystemic gradient was reduced to $10 \mathrm{mmHg}$ or less (16). Embolization of gastroesophageal varices was subsequently performed to reduce the flow of esophageal varices, thereby reducing the risk of ESD bleeding. All patients were admitted for at least one night for observation and had a complete blood count on postoperative day 1 .

ESD procedure. All ESD procedures were performed by the same endoscopist. The standard procedures were performed as previously reported (7). Briefly, the patients were maintained under conscious sedation using intravenous propofol, and their blood pressure, electrocardiogram and blood oxygen saturation values were monitored. An endoscope with a water jet system (cat. no. GIF-Q260J; Olympus Corporation) was used for the
ESD procedure with a transparent cap attached to its tip. A needle knife (cat. no. NM-400U-0423; Olympus Corporation), a hook knife (cat. no. KD-620 LR; Olympus Corporation), an insulated-tip knife-2 (cat. no. KD-611 L; Olympus Corporation) and a hemostatic forceps (cat no. FD-410 LR; Olympus Corporation) were used during the procedure. The electrosurgical generator used in the ESD was an ICC 200 device (Erbe Elektromedizin GmbH, Tuebingen, Germany) or a VIO300D device (Erbe Elektromedizin GmbH). The EEC lesions were detected with white light endoscopy, magnifying NBI and Lugol staining, as described previously (17), to estimate their depth and extent of invasion, and the lesions were marked by making spots with a needle-knife $\sim 5 \mathrm{~mm}$ outside the lesion. A mixture consisting of 30\% hyaluronic acid (Sigma-Aldrich; Merck KGaA, Darmstadt, Germany), 70\% saline solution (Chengdu KeLong Chemical Co., Ltd., Chengdu, China) and a small amount of indigo carmine (Micro-Tech, Co., Ltd., Nanjing, China) was subsequently injected into the submucosal layer to lift the lesion. Submucosal dissection was performed using a needle-knife, a hook knife or insulated tip knife-2. To control bleeding during the ESD procedure, hemostatic forceps were used in the soft coagulation mode (effect 5, 60 W). Procedure-associated bleeding after the ESD was defined as bleeding that required transfusion or surgical intervention or bleeding that caused the hemoglobin level to decrease by $2 \mathrm{~g} / \mathrm{dl}$ (18). Perforation was defined as detection of free air by a chest X-ray or computed tomography (CT) scan following the procedure. When EEC was located on an esophageal varix, endoscopic varix ligation (EVL) or TIPS was performed prior to the ESD procedure. ESD was performed one month following the EVL or TIPS procedure.

Radiofrequency ablation (RFA). Radiofrequency ablation was performed by the same endoscopist using a Barrx Flex RFA system (Covidien, Dublin, Ireland), as described previously (19). The system consisted of an ablation catheter, an energy generator and a sizing balloon. All RFA procedures were performed with the patients under conscious sedation or anesthesia. Prior to the RFA procedure, Lugol staining was performed to determine the location and size of the lesions. A radiant exposure of $12 \mathrm{~J} / \mathrm{cm}^{2}$ was used to minimize the risk of bleeding (14). Radiofrequency ablation was performed one month after the EVL or TIPS procedure.

Pathological analysis. ESD specimens were stretched, pinned on a styrofoam board and immediately immersed in $8 \%$ formalin at room temperature for $4 \mathrm{~h}$. Following fixation, the specimens were serially sectioned perpendicularly at $2-\mathrm{mm}$ intervals. Following deparaffinization and rehydration, $5-\mu \mathrm{m}$ longitudinal sections were stained with hematoxylin solution at room temperature for $5 \mathrm{~min}$ followed by five dips in $1 \%$ acid ethanol (1\% hydrocholoric acid in 70\% ethanol) and rinsed in distilled water. The sections were subsequently stained with eosin solution at room temperature for 3 min followed by dehydration with graded alcohol and clearing in xylene. The mounted slides were then examined and images were captured using an Olympus Corporation microscope. All specimens were reviewed by two gastrointestinal pathologists and the final pathological report was issued when the two pathologist agreed on the diagnosis. The tumor size, depth of invasion, 
Table I. Clinical characteristics of 6 patients with cirrhosis complicated by esophageal varices.

\begin{tabular}{lclcccccccc}
\hline Patient & Age & Etiology & $\begin{array}{c}\text { Child-Pugh } \\
\text { Class }\end{array}$ & INR & $\begin{array}{c}\text { Platelet } \\
\text { count, } \\
\text { x10 } / 1\end{array}$ & $\begin{array}{c}\text { Esophageal } \\
\text { varices }\end{array}$ & $\begin{array}{c}\text { EEC on } \\
\text { varices }\end{array}$ & $\begin{array}{c}\text { Lesion } \\
\text { morphology }\end{array}$ & $\begin{array}{c}\text { Lesion } \\
\text { length, cm }\end{array}$ & $\begin{array}{c}\text { Circumferential } \\
\text { extension }\end{array}$ \\
\hline 1 & 66 & HBV & A & 1.23 & 56 & F1,RC(-) & No & $0-$ IIb & 4 & $1 / 3$ \\
2 & 56 & HBV & B & 1.36 & 144 & F1,RC(-) & No & $0-$ Ilb & 2 & $1 / 3$ \\
3 & 48 & Alcohol & B & 1.29 & 63 & F2,RC(+) & Yes & $0-$ IIb & 6 & $3 / 4$ \\
4 & 66 & Alcohol & C & 1.35 & 40 & F1,RC(+) & Yes & $0-$ IIb & 3 & $1 / 2$ \\
5 & 53 & Alcohol & A & 1.12 & 45 & F2,RC(+) & Yes & $0-$ IIb & 6 & $3 / 5$ \\
6 & 52 & Alcohol & B & 1.51 & 86 & F2,RC(+) & Yes & $0-$ IIb & 5 & $3 / 4$ \\
\hline
\end{tabular}

HBV, hepatitis B virus; INR, international normalized ratio; EEC, early esophageal cancer, RC, red sign; F, form.

gross type, lymphatic and vascular involvement and tumor involvement of the lateral and vertical margins were assessed as described previously (7). Depth of invasion was classified into M1 (confined to the intraepithelium), M2 (confined to the lamina propria), M3 (confined to the muscularis mucosa), SM1 (submucosal invasion $<200 \mu \mathrm{m}$ ), and SM2\&3 (submucosal invasion $\geq 200 \mu \mathrm{m})(20)$.

Outcomes measurement and follow-up. During the procedure, the degree of intraoperative bleeding was defined as: Small bleeding, no obvious bleeding in the ESD procedure; medium bleeding, obvious bleeding which can be stopped immediately by endoscopy; large bleeding, hard to control or uncontrollable bleeding, or required blood transfusion following the procedure (21). Following the ESD or RFA procedure, intravenous administration of a proton pump inhibitor (omeprazole, $40 \mathrm{mg} / \mathrm{d}$ ) for two days and antibiotics (cefuroxime, $1.5 \mathrm{~g} / \mathrm{d}$ ) for three days was started on the day of the ESD or RFA procedure. The proton pump inhibitor was administered orally daily for two months thereafter. Blood cell counts, liver enzyme levels, bilirubin and albumin levels, international normalized ratio and $\mathrm{C}$-reactive protein level were assessed twice during the first week following the ESD as described previously (22). Follow-up endoscopy was performed two months following the ESD. The examinations were conducted every six months or every year thereafter. The last follow-up examination was conducted in July 2018.

\section{Results}

Clinical and endoscopic characteristics. The clinical and endoscopic characteristics of 6 patients with early EECs are presented in Table I. All patients had cirrhosis; 2 had hepatitis B virus cirrhosis and 4 had alcoholic cirrhosis. Of the 6 patients, five were Child-Pugh class $A$ and $B$, and one patient was Child-Pugh class $\mathrm{C}$. Three patients had $\mathrm{F} 1$ varices, and three had F2 varices (Table I). All EECs were flat with type IIb morphology. Four lesions were located overlying the esophageal varices, and two lesions (case 1 and case 2) were not close to the esophageal varices. The mean longitudinal length of the lesions was $4.3 \mathrm{~cm}$ (range, $2-6 \mathrm{~cm}$ ). All cases had a baseline diagnosis of high-grade dysplasia by biopsy prior to the procedure.
Outcomes and adverse events. The location of the EEC is presented in Table II. The average procedure time was $72.8 \mathrm{~min}$ (range, 34-135 min), and the average longitudinal length of the resected specimens was $53.0 \mathrm{~mm}$ (range, 30-90 mm). A total of 2 patients (cases 3 and 4) underwent EVL (representative image in Fig. S1), and 2 cases (cases 5 and 6) underwent TIPS to remove the varices prior to the treatment procedure as the EEC was located directly on the esophageal varices (Figs. 1 and 2). The remaining 2 cases (cases 1 and 2) underwent ESD directly without any additional treatment, as the lesions were not close to the esophageal varices (Table II; Fig. 3). Five patients underwent ESD and one case (case 6) underwent RFA due to diffuse 0-IIb lesions (Table II; Fig. 2). For ESD procedure, the en bloc resection rate was $100 \%(5 / 5$ lesions). Complete resection was defined as an en bloc resection with histologically confirmed tumor-free margins. En bloc and complete resection rates were $100 \%$ (6/6 lesions). Case 3 had tumor-positive lateral margins with lymphovascular infiltration (Table II). Therefore, the rates of both complete and curative resection were $80 \%$ (4/5 lesions).

Frequent intraoperative bleeding was detected in cases 3 and 4 undergoing EVL prior to ESD (Fig. S1). This bleeding was immediately stopped following several electrocoagulations, without causing life-threatening events. Nevertheless, less intraoperative bleeding, shorter operation time and fewer complications were present in patients undergoing TIPS prior to treatment (cases 5 and 6; Figs. 1 and 2). No severe complications or mortality-associated events, including massive postoperative bleeding, perforation or hepatic failure, were observed in any patient. Postoperative stricture occurred in one patient (case 3) due to the circumferential lesions (Fig. S1). Following three sessions of balloon dilation, the symptoms associated with the stricture were relieved in the follow-up period. The median follow-up period was 20 months. No recurrence and metastasis were observed during the follow-up period.

\section{Discussion}

Currently, patients with cirrhosis are considered to be at high risk for esophageal cancer (10). Studies reported that esophagectomy in patients with cirrhosis was associated with significant morbidity and mortality rates $(23,24)$. Patients with 
early esophageal cancer and cirrhosis are poor candidates for ESD or EMR due to increased procedure-associated complications (10). The current study investigated a treatment strategy for early esophageal cancers in patients with cirrhosis complicated by esophageal varices. For patients undergoing ESD or RFA procedures, the rates of en bloc and complete resections were satisfactory. No severe complications, including postprocedural bleeding or perforation, occurred and the safety profiles were satisfactory, suggesting that ESD or RFA could be safely applied for the treatment of early superficial esophageal cancer in patients with cirrhosis complicated by esophageal varices.

Previous studies reported successful ESD for patients with early EEC with esophageal varices, carrying a high risk of massive bleeding and requiring a high level of endoscopic expertise $(12,13)$. The risk of bleeding increased the lower the EEC, as the varicose veins flow mainly through inferior esophageal veins. Therefore, the lower location with much more esophageal veins had a higher risk of bleeding. Previous studies have reported the use of EMR or ESD for patients with EEC and cirrhosis combined with esophageal varices (25-27). The procedures performed relied on the removal of the esophageal varices using EVL or endoscopic injection sclerotherapy (EIS) (28). EIS requires considerable time to remove the esophageal varices and may induce submucosal fibrosis and scarring, possibly resulting in difficulty of submucosal lifting and incomplete resection (12). EVL was performed prior to ESD procedures in two cases (cases 3 and 4) in the current study. Intraprocedural bleeding occurred more significantly and frequently in the patients undergoing EVL procedures than in patients undergoing TIPS before ESD procedures. While several sessions of EVL may have reduced the size of the varices, they may have still been present during the ESD and may have resulted in bleeding (27). Furthermore, the timing of endoscopic treatment following EVL is important, and primarily determined by the size of esophageal varices (12). In the present study, if the esophageal varices were significantly reduced in size or removed, the ESD procedure was performed. If not, endoscopy examination was performed following one month to reevaluate the esophageal varices. In the present study, the esophageal varices were reduced in size or removed one month following EVL or TIPS. A previous study revealed that ulcers following EVL healed by 3 to 4 weeks, and the necrosis and fibrosis were limited to the mucosa and submucosa, with no involvement of the muscularis propria (28). Therefore, in the current study, the endoscopic treatment was performed one month following EVL or TIPS. One lesion (case 3) was not completely resected with tumor-positive margin. TIPS may therefore be superior to EVL for esophageal varices beneath the EECs because it provides effective relief of esophageal varices without submucosal adhesion or morphological changes prior to ESD or RFA procedures.

In the current study, the treatment regimens selected were primarily based on the morphological type and invasive depth of the lesions. EEC has three morphological types, including flat, degraded and elevated lesions (7). For widespread completely flat early EECs (type 0-IIb), RFA was used for the removal of EECs in the current study. Thus, assessing the depth of invasion and the morphological type is important. Prior to treatment, NBI-magnification endoscopy was used for endoscopic staging and endoscopic ultrasound was used for evaluating depth of 


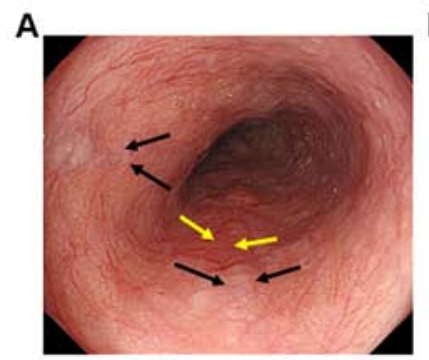

E

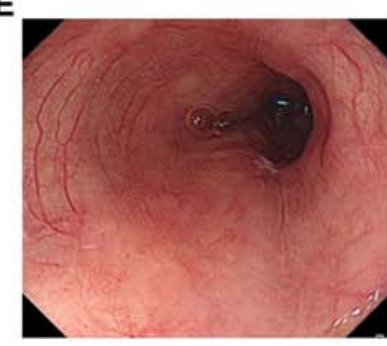

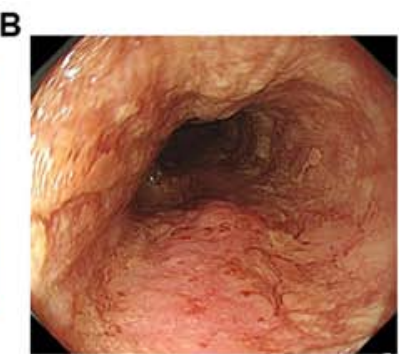

$\mathbf{F}$

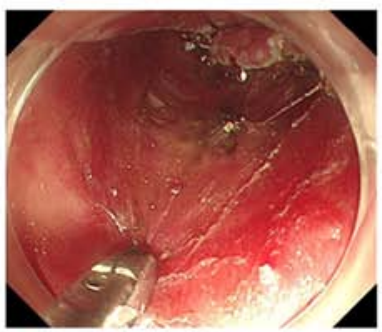

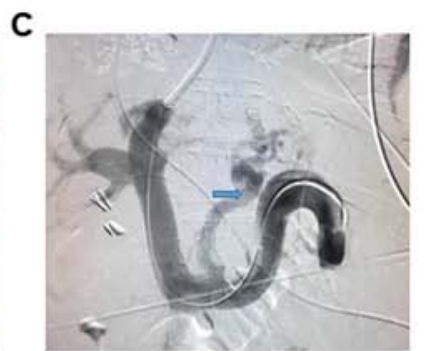

G

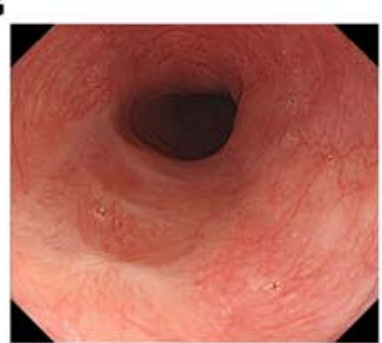

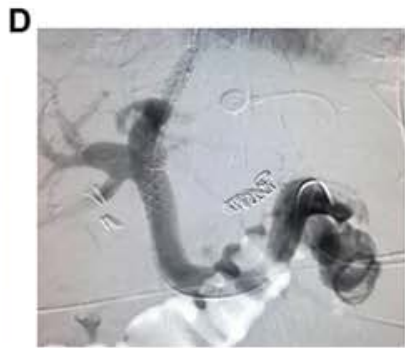

H

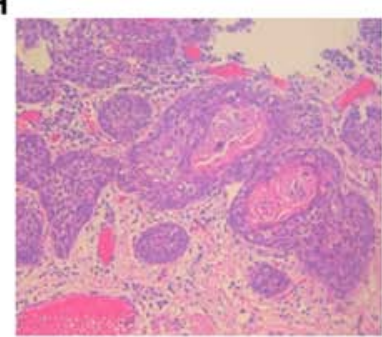

Figure 1. ESD for EEC concomitant with TIPS in case 5. (A) Irregular mucosa of superficial EEC (yellow arrows) located on a varix (black arrows) was observed by white light endoscopy. (B) The precise area of the EEC was determined with Lugol staining. (C) Radiological images before the stent implantation. The blue arrow indicates the varicose vein. (D) TIPS was performed prior to ESD and the radiological images presented are from after stent implantation. (E) White light endoscopy revealed no obvious esophageal varices near the lesion following TIPS. (F) ESD was completed successfully en bloc without any adverse events. (G) Near complete recovery of the epithelium with scarring one month following ESD. (H) A representative microscopic image of the resected tissues. Magnification, $\mathrm{x} 4$; scale bar= $250 \mu \mathrm{m}$; arrows indicate the area of interest. ESD, endoscopic submucosal dissection; EEC, early esophageal carcinoma; TIPS, transjugular intrahepatic portosystemic shunt.

A

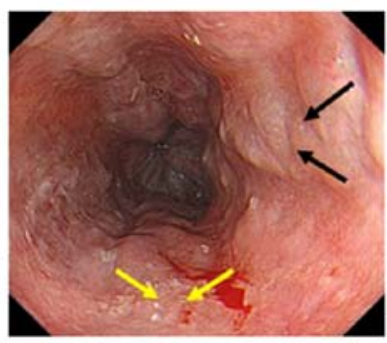

D

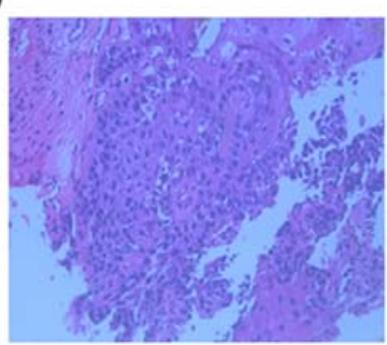

G

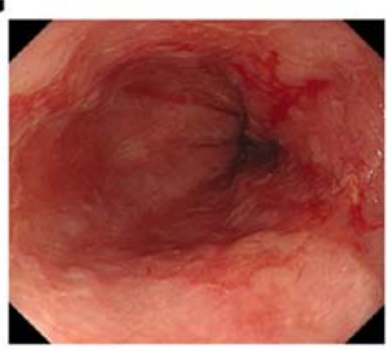

B

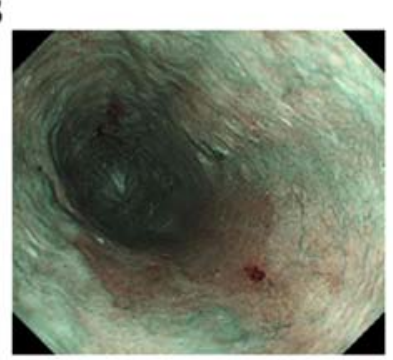

E

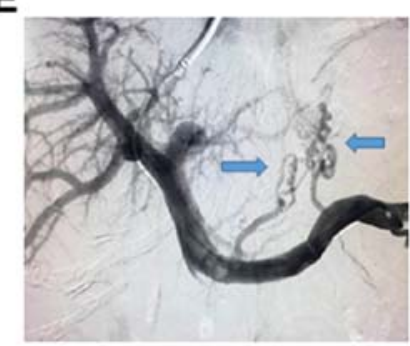

H

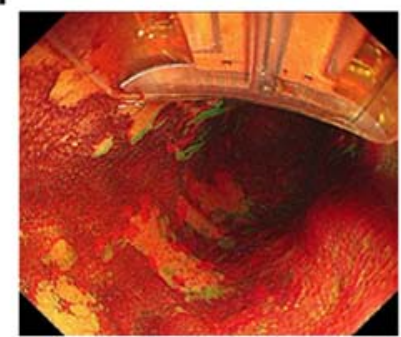

C

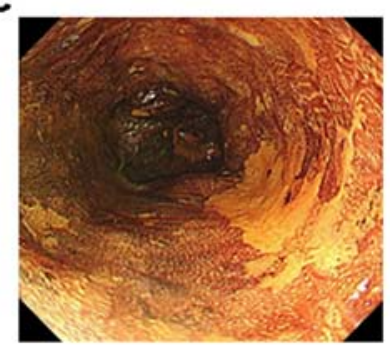

$\mathbf{F}$

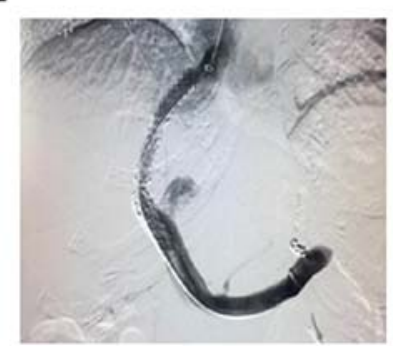

I

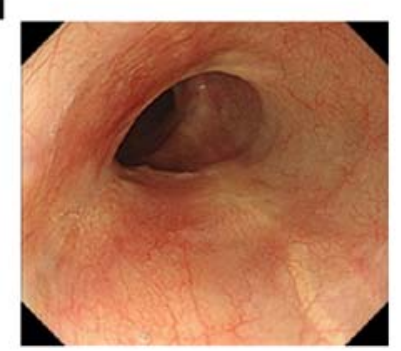

Figure 2. Representative patient (case 6) with early esophageal squamous neoplasia on esophageal varices treated with RFA. (A) White light imaging revealed a flat reddish lesion (yellow arrows) on esophageal varices (black arrows). (B) Narrow-band imaging revealed the brown area of the lesions. (C) The precise area of the early esophageal cancer was determined with Lugol staining. (D) A representative microscopic image of the lesions following biopsy. Magnification, $\mathrm{x} 4$; scale bar $=250 \mu \mathrm{m}$; arrows indicate the area of interest. (E) Radiological images before stent implantation. The blue arrows indicate the varicose vein. (F) TIPS was performed prior to RFA and the radiological images presented are from after stent implantation. (G) White light endoscopy revealed no obvious esophageal varices near the lesion following TIPS. (H) RFA was performed. (I) One month following endoscopic submucosal dissection, the epithelium had almost recovered with scarring. RFA, radiofrequency ablation; TIPS, transjugular intrahepatic portosystemic shunt. 

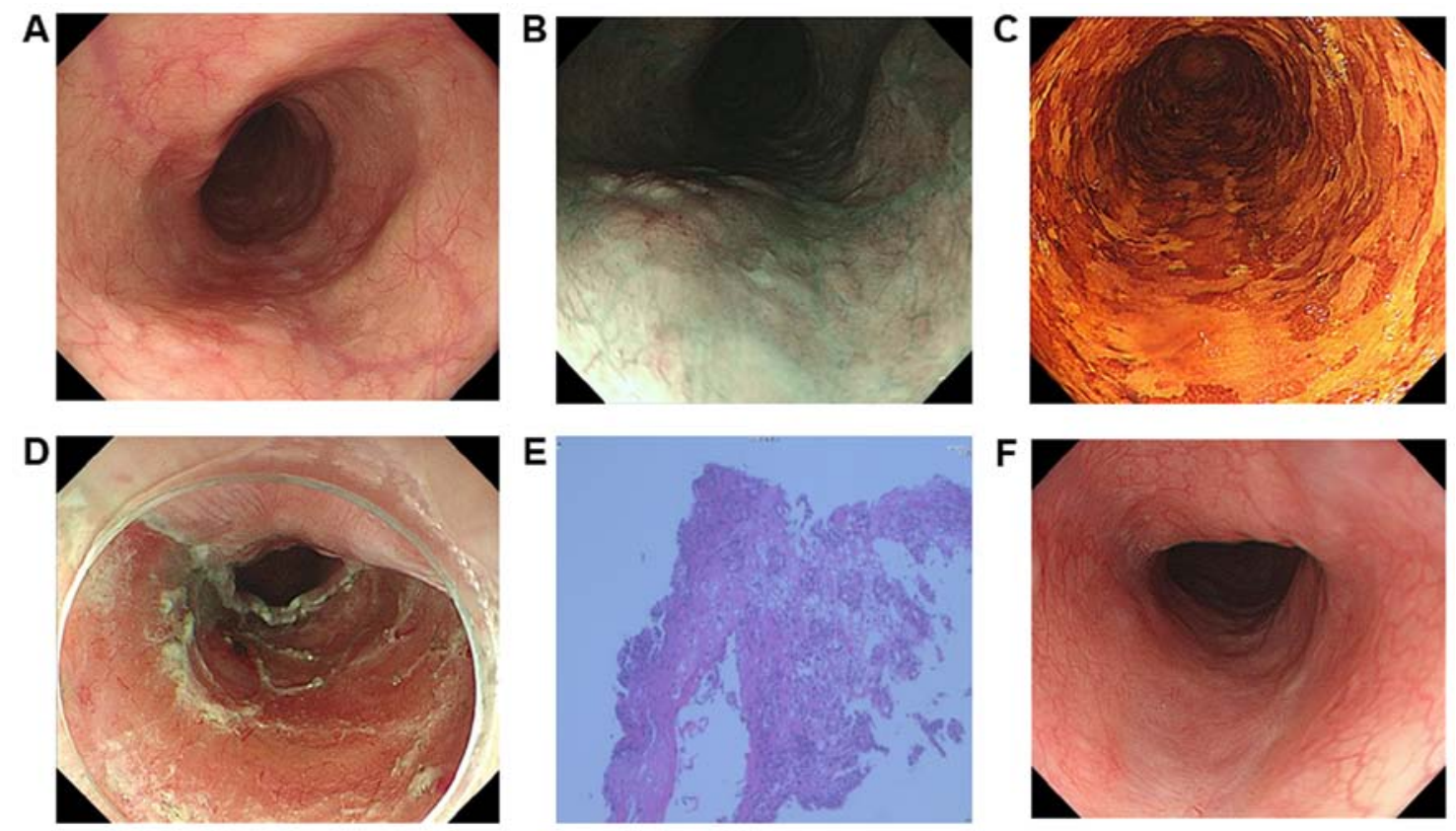

Figure 3. ESD for early esophageal carcinoma distant from the esophageal varices in case 2. (A) White light imaging revealed a reddish flat lesion (yellow arrows) distant from the esophageal varices (black arrows). (B) Narrow-band imaging revealed a brown area and dilation of intrapapillary capillary loop as depicted by the yellow arrows. (C) Lugol staining revealed the area of the lesion. (D) ESD was performed without massive intraoperative bleeding. (E) A microscopic image of the resected tissues Magnification, $\mathrm{x} 4$; scale bar=250 $\mu \mathrm{m}$; arrows indicate the area of interest. (F) One month following ESD, the epithelium recovered with scarring without stricture. ESD, endoscopic submucosal dissection.

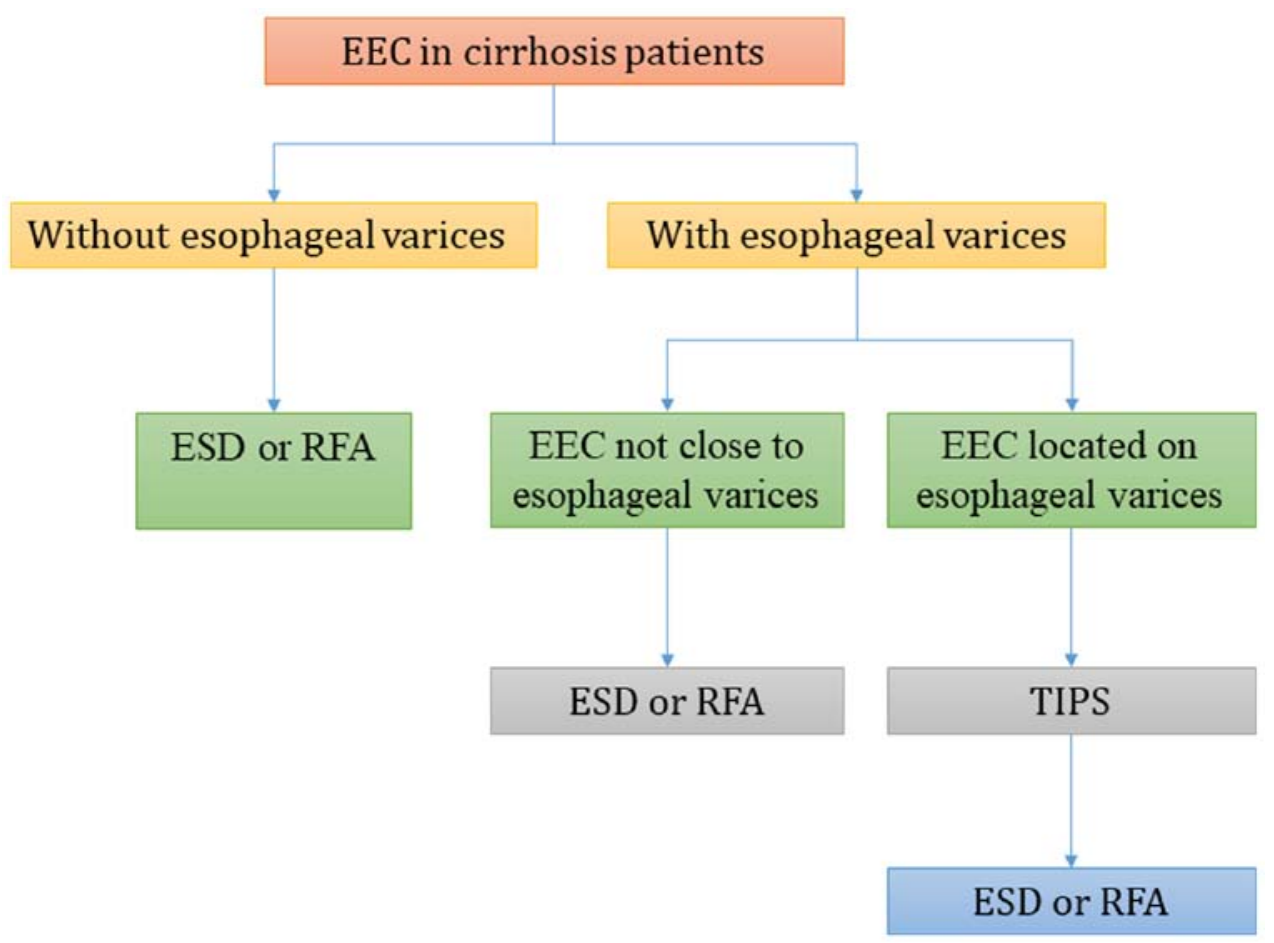

Figure 4. Flowchart of treatment of EEC in patients with cirrhosis. EEC, early esophageal carcinoma; ESD, endoscopic submucosal dissection; RFA, radiofrequency ablation; TIPS, transjugular intrahepatic portosystemic shunt.

invasion in the current study. Due to the limited cases, all six cases in our study had flat lesions. It is possible that all types of EECs were confronted with the similar risks of bleeding, and are therefore suitable for this treatment strategy (11-14).

The current study proposed a standard treatment workflow for EEC in patients with cirrhosis (Fig. 4). If there are no esophageal varices present in the context of cirrhosis, ESD or RFA may be performed without any preoperative treatment. Otherwise, the relative location of esophageal varices and the EEC should be further evaluated. If the EEC is located on the esophageal varix, TIPS is recommended to alleviate portal hypertension and relieve esophageal varices prior to ESD 
or RFA procedures. If the EEC is distant to the esophageal varices, further evaluation of the vertical depth of varices using CT scan or endoscopic ultrasonography is required. If the esophageal varices are located in the submucosa or muscle layer, TIPS is recommended to alleviate intraoperative bleeding as the submucosa is resected in the ESD procedure. ESD or RFA may be performed in other cases, dependent on the morphological and depth of invasion for EEC.

The major limitation of the present study was the small number of cases. Prospective multicenter studies are required to evaluate the efficacy and safety of the proposed treatment strategy. In conclusion, the present study described a novel treatment workflow for patients with EEC complicated by esophageal varices and cirrhosis. Good treatment results, no neoplastic progression and an acceptable adverse event profile were observed.

\section{Acknowledgements}

Not applicable.

\section{Funding}

No funding was received.

\section{Availability of data and materials}

All data generated or analyzed during this study are included in this published article.

\section{Authors' contributions}

SMY, BT, JY and ZGX conceived and designed the study. ZGX, YBZ and EL performed the analysis and interpretation of data. BT and ZGX drafted the manuscript. JYB performed the procedures and provided the samples. SMY and BT revised the manuscript. All authors approved the final version of the article.

\section{Ethics approval and consent to participate}

The present study was approved by The Ethics Committee of Xinqiao Hospital of Third Military Medical University (Chongqing, China). Informed consent for participation in this study was obtained from all patients.

\section{Patient consent for publication}

Not applicable

\section{Competing interests}

The authors declare that they have competing interests.

\section{References}

1. Patel V and Burbridge RA: Endoscopic approaches for early-stage esophageal cancer: Current options. Curr Oncol Rep 17: 421, 2015.

2. ASGE Standards of Practice Committee, Evans JA, Early DS, Chandraskhara V, Chathadi KV, Fanelli RD, Fisher DA, Foley KQ, Hwang JH, Jue TL, et al: The role of endoscopy in the assessment and treatment of esophageal cancer. Gastrointest Endosc 77: 328-334, 2013.
3. Lee CT, Chang CY, Lee YC, Tai CM, Wang WL, Tseng PH, Hwang JC, Hwang TZ, Wang CC and Lin JT: Narrow-band imaging with magnifying endoscopy for the screening of esophageal cancer in patients with primary head and neck cancers. Endoscopy 42: 613-619, 2010.

4. Barnes JA and Willingham FF: Endoscopic management of early esophageal cancer. J Clin Gastroenterol 49: 638-646, 2015.

5. Wang S, Huang Y, Xie J, Zhuge L, Shao L, Xiang J, Zhang Y, Sun Y, Hu H, Chen S, et al: Does delayed esophagectomy after endoscopic resection affect outcomes in patients with stage T1 esophageal cancer? A propensity score-based analysis. Surg Endosc 32: 1441-1448, 2018.

6. Ogura K, Okamoto M, Sugimoto T, Yahagi N, Fujishiro M, Kakushima N, Kodashima S, Kawabe T and Omata M: Efficacy and safety of endoscopic submucosal dissection for gastric cancer in patients with liver cirrhosis. Endoscopy 40: 443-445, 2008.

7. Tang B, Bai JY, Zhao XY, Fan CQ, Yang X, Deng L, Yang SM and Yu J: Endoscopic submucosal dissection for superficial esophageal cancer with near-circumferential lesions: Our experience with 40 patients. Surg Endosc 29: 2141-2148, 2015.

8. Kim JS, Kim BW and Shin IS: Efficacy and safety of endoscopic submucosal dissection for superficial squamous esophageal neoplasia: A meta-analysis. Dig Dis Sci 59: 1862-1869, 2014.

9. Basford PJ, George R, Nixon E, Chaudhuri T, Mead R and Bhandari P: Endoscopic resection of sporadic duodenal adenomas: Comparison of endoscopic mucosal resection (EMR) with hybrid endoscopic submucosal dissection (ESD) techniques and the risks of late delayed bleeding. Surg Endosc 28: 1594-1600, 2014

10. Ferro D, Angelico F, Caldwell SH and Violi F: Bleeding and thrombosis in cirrhotic patients: What really matters? Dig Liver Dis 44: 275-279, 2012.

11. Palmer WC, Di Leo M, Jovani M, Wolfsen HC, Krishna M and Wallace MB: Endoscopic management of high-grade dysplastic Barrett's esophagus with esophageal varices. Gastrointest Endosc 81: 997, 2015. Sawaguchi M, Jin M, Matsuhashi T, Ohba R, Hatakeyama N, Koizumi S, Onochi K, Yamada Y, Kanazawa N, Kimura Y, et al: The feasibility of endoscopic submucosal dissection for superficial esophageal cancer in patients with cirrhosis (with video). Gastrointest Endosc 79: 681-685, 2014

12. Hsu WH, Kuo CH, Wu IC, Lu CY, Wu DC and Hu HM: Superficial esophageal squamous cell carcinoma over esophageal varices treated by endoscopic submucosal dissection. Gastrointest Endosc 79: 833-834, 2014.

13. Wang WL, Chang IW, Chen CC, Chang CY, Mo LR, Lin JT, Wang HP and Lee CT: A case series on the use of circumferential radiofrequency ablation for early esophageal squamous neoplasias in patients with esophageal varices. Gastrointest Endosc 85: 322-329, 2017.

14. Lee E, Kim YJ, Goo DE, Yang SB, Kim HJ, Jang JY and Jeong SW: Comparison of hepatic venous pressure gradient and endoscopic grading of esophageal varices. World J Gastroenterol 22: 3212-3219, 2016

15. Verbeeck S, Mekhali D, Cassiman D, Maleux G and Witters P: Long-term outcome of transjugular intrahepatic portosystemic shunt for portal hypertension in autosomal recessive polycystic kidney disease. Dig Liver Dis 50: 707-712, 2018.

16. Heresbach D, Leray E, d'Halluin PN, Cholet F, Lapalus MG, Gaudric M, Ben Soussan E, Gaudin JL, Vahedi K, Quentin V, et al: Diagnostic accuracy of esophageal capsule endoscopy versus conventional upper digestive endoscopy for suspected esophageal squamous cell carcinoma. Endoscopy 42: 93-97, 2010.

17. Imagawa A, Okada $\mathrm{H}$, Kawahara $\mathrm{Y}$, Takenaka $\mathrm{R}$, Kato $\mathrm{J}$, Kawamoto H, Fujiki S, Takata R, Yoshino T and Shiratori Y: Endoscopic submucosal dissection for early gastric cancer: Results and degrees of technical difficulty as well as success. Endoscopy 38: 987-990, 2006.

18. Chen WC and Wolfsen H: Role of radiofrequency ablation in esophageal squamous dysplasia and early neoplasia. Gastrointest Endosc 85: 330-331, 2017.

19. Ishihara R, Matsuura N, Hanaoka N, Yamamoto S, Akasaka T, Takeuchi Y, Higashino K, Uedo N and Iishi H: Endoscopic imaging modalities for diagnosing invasion depth of superficial esophageal squamous cell carcinoma: A systematic review and meta-analysis. BMC Gastroenterol 17: 24, 2017.

20. Kataoka Y,Tsuji Y, Sakaguchi Y, Minatsuki C, Asada-Hirayama I, Niimi K, Ono S, Kodashima S, Yamamichi N, Fujishiro M and Koike K: Bleeding after endoscopic submucosal dissection: Risk factors and preventive methods. World J Gastroenterol 22: 5927-5935, 2016 
21. Tsou YK, Liu CY, Fu KI, Lin CH, Lee MS, Su MY, Ohata K and Chiu CT: Endoscopic submucosal dissection of superficial esophageal neoplasms is feasible and not riskier for patients with liver cirrhosis. Dig Dis Sci 61: 3565-3571, 2016.

22. Mariette C: Is there a place for esogastric cancer surgery in cirrhotic patients? Ann Surg Oncol 15: 680-682, 2008.

23. Asti E, Sozzi M, Bonitta G, Bernardi D and Bonavina L: Esophagectomy in patients with liver cirrhosis: A systematic review and Bayesian meta-analysis. J Visc Surg 155: 453-464, 2018.

24. Inoue H, Endo M, Takeshita K, Shimoju K, Yoshino K, Goseki N and Sasabe M: Endoscopic resection of carcinoma in situ of the esophagus accompanied by esophageal varices. Surg Endosc 5: 182-184, 1991

25. Jovanovic I, Krivokapic Z, Menkovic N, Krstic M and Mönkemüller K: Ineffectiveness of capsule endoscopy and total double-balloon enteroscopy to elicit the cause of obscure overt gastrointestinal bleeding: Think GIST! Endoscopy 43 (Suppl 2) UCTN: E91-E92, 2011.
26. Künzli HT and Weusten BL: Endoscopic resection of early esophageal neoplasia in patients with esophageal varices: How to succeed while preventing the bleed. Endoscopy 46 (Suppl 1) UCTN: E631-E632, 2014

27. Iwase H, Kusugami K, Suzuki M, Nishio Y, Ando T, Ina K and Peek RM: Endoscopic resection of early-stage esophageal cancer accompanied by esophageal varices. Gastrointest Endosc 51: 749-752, 2000

28. Polski JM, Brunt EM and Saeed ZA: Chronology of histological changes after band ligation of esophageal varices in humans. Endoscopy 33: 443-447, 2001. 